\title{
The Solutions of Mixed Monotone Fredholm-Type Integral Equations in Banach Spaces
}

\author{
Hua Su \\ School of Mathematics and Quantitative Economics, Shandong University of Finance and Economics, Jinan, Shandong 250014, China \\ Correspondence should be addressed to Hua Su; jnsuhua@163.com \\ Received 15 January 2013; Accepted 23 February 2013 \\ Academic Editor: Yanbin Sang \\ Copyright (C) 2013 Hua Su. This is an open access article distributed under the Creative Commons Attribution License, which \\ permits unrestricted use, distribution, and reproduction in any medium, provided the original work is properly cited. \\ By introducing new definitions of $\phi$ convex and $-\varphi$ concave quasioperator and $v_{0}$ quasilower and $u_{0}$ quasiupper, by means of \\ the monotone iterative techniques without any compactness conditions, we obtain the iterative unique solution of nonlinear mixed \\ monotone Fredholm-type integral equations in Banach spaces. Our results are even new to $\phi$ convex and $-\varphi$ concave quasi operator, \\ and then we apply these results to the two-point boundary value problem of second-order nonlinear ordinary differential equations \\ in the ordered Banach spaces.
}

\section{Introduction}

In this paper, we will consider the following nonlinear Fredholm integral equation:

$$
u(t)=\int_{I} H(t, s, u(s)) d s, \quad t \in I,
$$

where $I=[a, b]$ and $H \in C[I \times I \times E, E], E$ is a real Banach space with the norm $\|\cdot\|$, and there exists a function $G \in$ $C[I \times I \times E \times E, E]$ such that for any $(t, s, x) \in I \times I \times E$

$$
H(t, s, x)=G(t, s, x, x) .
$$

Guo and Lakshmikantham [1] introduced the definition of mixed monotone operator and coupled fixed point; there are many good results (see [1-13]). In the special case where $H(t, s, x)$ is nondecreasing in $x$ for fixed $t, s \in I$, Guo [2] established an existence theorem of the maximal and minimal solutions for (1) in the ordered Banach spaces by means of monotone iterative techniques. Recently, Jingxian and Lishan [3] and Lishan [4] obtained iterative sequences that converge uniformly to solutions and coupled minimal and maximal quasisolutions of the nonlinear Fredholm integral equations in ordered Banach spaces by using the Möuch fixed point theorem and establishing new comparison results. But these all required the compactness conditions and the monotone conditions in the above papers, and furthermore they did not obtain the unique solutions. In addition, extensive studies have also been carried out to study the global or iterative solutions of initial value problems [8-13].

In this paper, by introducing new definitions of $\phi$ convex and $-\varphi$ concave quasioperator and $v_{0}$ quasilower and $u_{0}$ quasiupper, by means of the monotone iterative techniques without any compactness conditions which are of the essence in $[2-4,7,8,14]$, we obtain the iterative unique solution of nonlinear mixed monotone Fredholm-type integral equations in Banach spaces and then apply these results to the twopoint boundary value problem of second-order nonlinear ordinary differential equations.

\section{Preliminaries and Definitions}

Let $P$ be a cone in $E$, that is, a closed convex subset such that $\lambda P \subset P$ for any $\lambda \geq 0$ and $P \cap\{-P\}=\{\theta\}$. By means of $P$, a partial order $\leq$ is defined as $x \leq y$ if and only if $y-x \in P$. A cone $P$ is said to be normal if there exists a constant $N>$ 0 such that $x, y \in E, \theta \leq x \leq y$ implies $\|x\| \leq N\|y\|$, where $\theta$ denotes the zero element of $E$ (see $[2,14])$, and we call the smallest number $N$ the normal constant of $P$ and denote $N_{P}$. The cone $P$ is normal if and only if every ordered interval $[x, y]=\{z \in E: x \leq z \leq y\}$ is bounded.

Let $P_{I}=\{u \in C[I, E]: u(t) \geq \theta$ for all $t \in I\}$, where $C[I, E]$ denotes the Banach space of all the continuous 
mapping $u: I \rightarrow E$ with the norm $\|u\|_{C}=\max _{t \in I}|u(t)|$. It is clear that $P_{I}$ is a cone of space $C[I, E]$, and so it defines a partial ordering in $C[I, E]$. Obviously, the normality of $P$ implies the normality of $P_{I}$ and the normal constants of $P_{I}$, and $P$ are the same.

Let $u_{0}, v_{0} \in C[I, E]$. Then, $u_{0}, v_{0}$ are said to be coupled lower and upper quasi-solutions of (1) if

$$
\begin{aligned}
& u_{0}(t) \leq \int_{I} G\left(t, s, u_{0}(s), v_{0}(s)\right) d s, \quad t \in I, \\
& v_{0}(t) \geq \int_{I} G\left(t, s, v_{0}(s), u_{0}(s)\right) d s, \quad t \in I .
\end{aligned}
$$

If the equality in (3) holds, then $u_{0}, v_{0}$ are said to be coupled quasi-solutions of (1).

We will always assume in this paper that $P$ is a normal cone of $E$. For any $u_{0}, v_{0} \in C[I, E]$ such that $v_{0} \leq w_{0}$, we define the ordered interval $D=\left[u_{0}, v_{0}\right]=\left\{u \in C[I, E]: u_{0} \leq\right.$ $\left.u \leq v_{0}\right\}$.

Next, we will give the new definition of $\phi$ convex and $-\varphi$ concave quasi operator and $v_{0}$ quasi-lower and $u_{0}$ quasiupper.

Definition 1. Suppose that, $G \in C[I \times I \times E \times E, E]$. Then $G$ is called $\phi$ convex and $-\varphi$ concave quasi operator, if there exist functions

$$
\begin{aligned}
& \phi:(0, \infty) \times(0, \infty) \longrightarrow(0, \infty), \\
& \varphi:(0, \infty) \times(0, \infty) \longrightarrow(0, \infty),
\end{aligned}
$$

such that

(1) $G(t, s, \alpha u, \beta v) \geq \phi(\alpha, \beta) G(t, s, u, v), \alpha<\beta, \alpha, \beta \epsilon$ $(0, \infty)$, for all $u, v \in E$,

(2) $G(t, s, \alpha u, \beta v) \leq \varphi(\alpha, \beta) G(t, s, u, v), \alpha \geq \beta, \alpha, \beta \epsilon$ $(0, \infty)$, for all $u, v \in E$.

Definition 2. Suppose that $G \in C[I \times I \times E \times E, E], u_{0} \in P$. Then, $G$ is called $u_{0}$ quasi-upper, if for any $u, v \in E, u, v<u_{0}$ such that $\int_{I} G(t, s, u, v) d s<u_{0}$.

Definition 3. Suppose that $G \in C[I \times I \times E \times E, E], v_{0} \in E$. Then, $G$ is called $v_{0}$ quasi-lower, if for any $u, v \in E, u, v>v_{0}$ such that $\int_{I} G(t, s, u, v) d s>v_{0}$.

Let us list the following assumption for convenience.

$\left(H_{1}\right) G$ is uniformly continuous on $I \times I \times E \times E$, and $G$ is $\phi$ convex and $-\varphi$ concave quasi operator.

$\left(H_{2}\right) G(t, s, x, y)$ is nondecreasing in $x \in E$ for fixed $(t, s, y) \in I \times I \times E . G(t, s, x, y)$ is nonincreasing in $y \in E$ for fixed $(t, s, x) \in I \times I \times E$.

$\left(H_{3}\right) \phi(\alpha, \beta), \varphi(\alpha, \beta)$ are all increasing in $\alpha$, decreasing in $\beta$, and $\phi\left(\alpha_{0}, \beta_{0}\right) \geq \alpha_{0}, \varphi\left(\beta_{0}, \alpha_{0}\right) \leq \beta_{0}$ and for $\alpha, \beta \in$ $\left[\alpha_{0}, \beta_{0}\right], \alpha<\beta$,

$$
\varphi(\beta, \alpha)-\phi(\alpha, \beta) \leq l(\beta-\alpha), \quad 0<l<1 .
$$

\section{The Main Result}

The main results of this paper are the following three theorems.

Theorem 4. Let $P$ be a normal cone of $E$, let $u_{0}, v_{0} \in P_{I}$ be coupled lower and upper quasi-solutions of (1). Assume that conditions $\left(H_{1}\right),\left(H_{2}\right)$, and $\left(H_{3}\right)$ hold and

$\left(H_{4}\right)$ There exists $w_{0} \in P_{I}$ such that $u_{0} \leq w_{0} \leq v_{0}$, and for $\alpha_{0}, \beta_{0} \in(0, \infty)$ of $\left(H_{3}\right)$ such that $u_{0} \geq \alpha_{0} w_{0}, \beta_{0} w_{0} \geq$ $v_{0}$.

Then, (1) has a unique solution $x^{*}(t) \in D=\left[u_{0}, v_{0}\right]$, and for any initial $x_{0}, y_{0} \in\left[u_{0}, v_{0}\right]$, one has

$$
\begin{gathered}
x_{n}(t) \longrightarrow x^{*}(t), \quad y_{n}(t) \longrightarrow x^{*}(t), \\
\text { uniformly on } t \in I \quad \text { as } n \longrightarrow \infty,
\end{gathered}
$$

where $\left\{x_{n}(t)\right\},\left\{y_{n}(t)\right\}$ are defined as

$$
\begin{gathered}
x_{n}(t)=\int_{I} G\left(t, s, x_{n-1}(s), y_{n-1}(s)\right) d s, \\
y_{n}(t)=\int_{I} G\left(t, s, y_{n-1}(s), x_{n-1}(s)\right) d s, \quad t \in I .
\end{gathered}
$$

Proof. We first define the operator $A:\left[u_{0}, v_{0}\right] \times\left[u_{0}, v_{0}\right] \rightarrow$ $C[I, E]$ by the formula

$$
A(u, v)=\int_{I} G(t, s, u(s), v(s)) d s
$$

It follows from the assumption $\left(H_{2}\right)$ that $A$ is a mixed monotone operator, that is, $A(u, v)$ is nondecreasing in $u \in$ $\left[u_{0}, v_{0}\right]$ and nonincreasing in $v \in\left[u_{0}, v_{0}\right]$, and $u_{0} \leq$ $A\left(u_{0}, v_{0}\right), A\left(v_{0}, u_{0}\right) \leq v_{0}$.

By (7), we have $x_{n}(t)=A\left(x_{n-1}(t), y_{n-1}(t)\right), y_{n}(t)=$ $A\left(y_{n-1}(t), x_{n-1}(t)\right)$ and set $w_{n}(t)=A\left(w_{n-1}(t), w_{n-1}(t)\right)$ for initial $w_{0}$ in $\left(H_{4}\right)$, and we also define that

$$
\begin{aligned}
& u_{n}(t)=A\left(u_{n-1}(t), v_{n-1}(t)\right), \\
& v_{n}(t)=A\left(v_{n-1}(t), u_{n-1}(t)\right) .
\end{aligned}
$$

Since $A$ is a mixed monotone operator, it is easy to see that

$$
\begin{array}{r}
u_{0} \leq u_{1} \leq \cdots \leq u_{n} \leq \cdots \leq v_{n} \leq \cdots \leq v_{1} \leq v_{0} \\
u_{n} \leq w_{n} \leq v_{n} .
\end{array}
$$

Obviously, by induction, it is easy to see that

$$
\begin{gathered}
u_{n} \geq \alpha_{n} w_{n}, \quad v_{n} \leq \beta_{n} w_{n}, \quad n=0,1, \ldots, \\
\alpha_{0} \leq \alpha_{1} \leq \cdots \leq \alpha_{n} \leq \cdots \leq 1 \leq \cdots \leq \beta_{n} \leq \cdots \leq \beta_{1} \leq \beta_{0},
\end{gathered}
$$

where $\alpha_{n}=\phi\left(\alpha_{n-1}, \beta_{n-1}\right), \beta_{n}=\varphi\left(\beta_{n-1}, \alpha_{n-1}\right), n=1,2, \ldots$

In fact, by the assumption $\left(H_{4}\right)$, we have that inequality (11) holds as $n=0$. Suppose that inequality (11) holds as $n=k$, 
that is, $u_{k} \geq \alpha_{k} w_{k}, v_{k} \leq \beta_{k} w_{k}$. Then, as $n=k+1$, by the assumption $\left(H_{3}\right)$, we have

$$
\begin{aligned}
u_{k+1} & =A\left(u_{k}, v_{k}\right)=\int_{I} G\left(t, s, u_{k}(s), v_{k}(s)\right) d s \\
& \geq \int_{I} G\left(t, s, \alpha_{k} w_{k}, \beta_{k} w_{k}\right) d s \\
& \geq \phi\left(\alpha_{k}, \beta_{k}\right) \int_{I} G\left(t, s, w_{k}(s), w_{k}(s)\right) d s=\alpha_{k+1} w_{k+1}, \\
v_{k+1} & =A\left(v_{k}, u_{k}\right)=\int_{I} G\left(t, s, v_{k}(s), u_{k}(s)\right) d s \\
& \leq \int_{I} G\left(t, s, \beta_{k} w_{k}, \alpha_{k} w_{k}\right) d s \\
& \leq \varphi\left(\beta_{k}, \alpha_{k}\right) \int_{I} G\left(t, s, w_{k}(s), w_{k}(s)\right) d s=\beta_{k+1} w_{k+1} .
\end{aligned}
$$

Then, it is easy to show by induction that inequality (11) holds.

For inequality (12), by $u_{k+1} \leq w_{k+1} \leq v_{k+1}$ and the above discussion, we have $0<\alpha_{k+1} \leq 1 \leq \beta_{k+1}$. Obviously, it follows from the assumption $\left(H_{4}\right)$ that $\alpha_{0} \leq \alpha_{1}, \beta_{1} \leq \beta_{0}$. Suppose that $\alpha_{k-1} \leq \alpha_{k}, \beta_{k} \leq \beta_{k-1}$, so it is easy to show by $\left(H_{3}\right)$ that

$$
\begin{aligned}
& \phi\left(\alpha_{k-1}, \beta_{k-1}\right) \leq \phi\left(\alpha_{k}, \beta_{k}\right), \\
& \varphi\left(\beta_{k}, \alpha_{k}\right) \leq \varphi\left(\beta_{k-1}, \alpha_{k-1}\right),
\end{aligned}
$$

that is, $\alpha_{k} \leq \alpha_{k+1}, \beta_{k+1} \leq \beta_{k}$. Then, it is easy to show by induction that inequality (12) holds.

Then, it follows from the inequality (12) that there exist limits of the sequences $\left\{\alpha_{n}\right\},\left\{\beta_{n}\right\}$. Suppose that there exist $\alpha, \beta$ such that $\alpha_{n} \rightarrow \alpha, \beta_{n} \rightarrow \beta$, and $n \rightarrow \infty$, and by $\left(H_{3}\right)$, we also have

$$
\begin{aligned}
0 \leq \beta_{n}-\alpha_{n} & =\varphi\left(\beta_{n-1}, \alpha_{n-1}\right)-\phi\left(\alpha_{n-1}, \beta_{n-1}\right) \\
& \leq l\left(\beta_{n-1}-\alpha_{n-1}\right) \leq \cdots \leq l^{n}\left(\beta_{0}-\alpha_{0}\right),
\end{aligned}
$$

they $0<l<1$, and taking limits in the above inequality as $n \rightarrow \infty$, we have $\alpha=\beta$.

Next, we will show that the sequences $\left\{u_{n}\right\},\left\{v_{n}\right\}$ are all Cauchy sequences on $D$.

In fact, by (10) and (11), for any natural number $p$, we know that

$$
\begin{aligned}
& \theta \leq u_{n+p}-u_{n} \leq v_{n}-u_{n} \leq\left(\beta_{n}-\alpha_{n}\right) u_{0}, \\
& \theta \leq v_{n}-v_{n+p} \leq v_{n}-u_{n} \leq\left(\beta_{n}-\alpha_{n}\right) u_{0} .
\end{aligned}
$$

By the normality of $P_{I}$ and (15), we have

$$
\begin{aligned}
& \left\|u_{n+p}-u_{n}\right\|_{C} \leq N_{P} l^{n}\left(\beta_{0}-\alpha_{0}\right)\left\|u_{0}\right\|_{C}, \\
& \left\|v_{n}-v_{n+p}\right\|_{C} \leq N_{P} l^{n}\left(\beta_{0}-\alpha_{0}\right)\left\|u_{0}\right\|_{C},
\end{aligned}
$$

where $N_{P}$ is a normal constant. So $\left\{u_{n}\right\},\left\{v_{n}\right\}$ are all Cauchy sequences on $D$, and then there exists $u^{*}, v^{*} \in\left[u_{0}, v_{0}\right]$ such that $\lim _{n \rightarrow \infty} u_{n}=u^{*}, \lim _{n \rightarrow \infty} v_{n}=v^{*}$.
It is easy to know by (10) and (11) that

$\theta \leq v_{n}-u_{n} \leq \beta_{n} w_{n}-\alpha_{n} w_{n} \leq\left(\beta_{n}-\alpha_{n}\right) u_{0} \leq l^{n}\left(\beta_{0}-\alpha_{0}\right) u_{0}$,

so by the normality of $P_{I}$, we have

$$
\left\|v_{n}-u_{n}\right\|_{C} \leq N_{P} l^{n}\left(\beta_{0}-\alpha_{0}\right)\left\|u_{0}\right\|_{C}
$$

and taking limits in the above inequality as $n \rightarrow \infty$, we have $x^{*}=u^{*}=v^{*} \in\left[u_{0}, v_{0}\right]$, and for any natural number $n$, we also have $u_{n} \leq x^{*} \leq v_{n}, t \in I$.

Then, by the mixed monotone quality of $A$ we have

$$
u_{n+1}=A\left(u_{n}, v_{n}\right) \leq A\left(x^{*}, x^{*}\right) \leq A\left(v_{n}, u_{n}\right)=v_{n+1},
$$

and taking limits in above inequality as $n \rightarrow \infty$, we know that

$$
x^{*}=A\left(x^{*}, x^{*}\right),
$$

that is, $x^{*} \in\left[u_{0}, v_{0}\right]$ is the fixed point of $A$; thus, $x^{*}$ is the solution of (1) on $D=\left[u_{0}, v_{0}\right]$.

Furthermore, we will show that the solution is unique. Suppose that $y^{*} \in\left[u_{0}, v_{0}\right]$ satisfy $y^{*}=A\left(y^{*}, y^{*}\right)$. Then, by the mixed monotone quality of $A$ and induction, for any natural number $n$, it is easy to have that $u_{n} \leq y^{*} \leq v_{n}$. Then, by the normality of $P_{I}$ and taking limits in the above inequality as $n \rightarrow \infty$ and the above discussion, we have $y^{*}=x^{*}$.

For any initial $x_{0}, y_{0} \in\left[u_{0}, v_{0}\right]$, by (7) and (8), the mixed monotone quality of $A$ and induction, for any natural number $n$, we have $u_{n}(t) \leq x_{n}(t) \leq v_{n}(t), u_{n}(t) \leq y_{n}(t) \leq v_{n}(t), t \in I$. Then, the normality of $P_{I}$ and (19) imply that

$$
\begin{gathered}
\left\|x_{n}-u_{n}\right\|_{C} \leq N_{P} l^{n}\left(\beta_{0}-\alpha_{0}\right)\left\|u_{0}\right\|_{C}, \\
\left\|y_{n}-u_{n}\right\|_{C} \leq N_{P} N_{P} l^{n}\left(\beta_{0}-\alpha_{0}\right)\left\|u_{0}\right\|_{C} .
\end{gathered}
$$

Thus, the sequence $\left\{x_{n}(t)\right\},\left\{y_{n}(t)\right\}$ all converges uniformly to $x^{*}(t)$ on $t \in I$. This completes the proof of Theorem 4 .

Theorem 5. Let $P$ be a normal cone of $E$, let $u_{0}, v_{0} \in P_{I}$ be coupled lower and upper quasi-solutions of (1). Assume that conditions $\left(H_{1}\right),\left(H_{2}\right)$, and $\left(H_{3}\right)$ hold.

$\left(H_{4}^{\prime}\right) G$ is $u_{0}$ quasi-upper, and there exists $w_{0} \in P_{I}$ such that $w_{0}<u_{0}<v_{0}$, and there exist $\alpha_{0}=\sup \left\{\alpha>0: u_{0} \geq\right.$ $\left.\alpha w_{0}\right\}, \beta_{0}=\inf \left\{\beta>0: v_{0} \leq \beta w_{0}\right\}$.

Then, (1) has a unique solution $x^{*}(t) \in D=\left[u_{0}, v_{0}\right]$, and for any initial $x_{0}, y_{0} \in\left[u_{0}, v_{0}\right]$, one has

$$
x_{n}(t) \longrightarrow x^{*}(t), \quad y_{n}(t) \longrightarrow x^{*}(t),
$$

uniformly on $t \in I$ as $n \longrightarrow \infty$,

where $\left\{x_{n}(t)\right\},\left\{y_{n}(t)\right\}$ are defined as

$$
\begin{gathered}
x_{n}(t)=\int_{I} G\left(t, s, x_{n-1}(s), y_{n-1}(s)\right) d s, \\
y_{n}(t)=\int_{I} G\left(t, s, y_{n-1}(s), x_{n-1}(s)\right) d s, \quad t \in I .
\end{gathered}
$$


Proof. We first define the operator $A:\left[u_{0}, v_{0}\right] \times\left[u_{0}, v_{0}\right] \rightarrow$ $C[I, E]$ by the formula

$$
A(u, v)=\int_{I} G(t, s, u(s), v(s)) d s
$$

It follows from the assumption $\left(H_{2}\right)$ that $A$ is a mixed monotone operator, that is, $A(u, v)$ is nondecreasing in $u \in$ $\left[u_{0}, v_{0}\right]$ and nonincreasing in $v \in\left[u_{0}, v_{0}\right]$ and $u_{0} \leq$ $A\left(u_{0}, v_{0}\right), A\left(v_{0}, u_{0}\right) \leq v_{0}$. By $(7)$, we have $x_{n}(t)=$ $A\left(x_{n-1}(t), y_{n-1}(t)\right), y_{n}(t)=A\left(y_{n-1}(t), x_{n-1}(t)\right)$ and set $w_{n}(t)=A\left(w_{n-1}(t), w_{n-1}(t)\right)$, and we also define

$$
\begin{aligned}
& u_{n}(t)=A\left(u_{n-1}(t), v_{n-1}(t)\right), \\
& v_{n}(t)=A\left(v_{n-1}(t), u_{n-1}(t)\right) .
\end{aligned}
$$

Since $A$ is a mixed monotone operator, it is easy to see that

$$
u_{0} \leq u_{1} \leq \cdots \leq u_{n} \leq \cdots \leq v_{n} \leq \cdots \leq v_{1} \leq v_{0}
$$

Because $G$ is $u_{0}$ quasi-upper and $w_{0}<u_{0}$, we have

$$
\begin{aligned}
w_{1}(t) & =A\left(w_{0}(t), w_{0}(t)\right) \\
& =\int_{I} G\left(t, s, w_{0}(s), w_{0}(s)\right) d s<u_{0} .
\end{aligned}
$$

So for any natural number $n$, by induction, we know that $w_{n}(t)=A\left(w_{n-1}(t), w_{n-1}(t)\right)<u_{0}$.

It is easy to see by induction that

$$
\begin{gathered}
u_{k} \geq \alpha_{k} w_{k}, \quad v_{k} \leq \beta_{k} w_{k}, \\
a_{0} \leq a_{1} \leq \cdots \leq a_{k} \leq \cdots \leq b_{k} \leq \cdots \leq b_{1} \leq b_{0},
\end{gathered}
$$

where $\alpha_{k}=\phi\left(\alpha_{k-1}, \beta_{k-1}\right), \beta_{k}=\varphi\left(\beta_{k-1}, \alpha_{k-1}\right), k=1,2, \ldots$

In fact, by the assumptions $\left(H_{1}\right)$ and $\left(H_{3}\right)$ and the above discussion, as $n=0$, we have

$$
\begin{aligned}
u_{1} & =A\left(u_{0}, v_{0}\right)=\int_{I} G\left(t, s, u_{0}(s), v_{0}(s)\right) d s \\
& \geq \int_{I} G\left(t, s, \alpha_{0} w_{0}, \beta_{0} w_{0}\right) d s \\
& \geq \phi\left(\alpha_{0}, \beta_{0}\right) \int_{I} G\left(t, s, w_{0}(s), w_{0}(s)\right) d s=\alpha_{1} w_{1}, \\
v_{1} & =A\left(v_{0}, u_{0}\right)=\int_{I} G\left(t, s, v_{0}(s), u_{0}(s)\right) d s \\
& \leq \int_{I} G\left(t, s, \beta_{0} w_{0}, \alpha_{0} w_{0}\right) d s \\
& \leq \varphi\left(\beta_{0}, \alpha_{0}\right) \int_{I} G\left(t, s, w_{0}(s), w_{0}(s)\right) d s=\beta_{1} w_{1} .
\end{aligned}
$$

By the above two inequalities and assumption $\left(H_{3}\right)$, we have

$$
a_{0} \leq \alpha_{1}=\phi\left(\alpha_{0}, \beta_{0}\right) \leq \varphi\left(\beta_{0}, \alpha_{0}\right)=\beta_{1} \leq b_{0} .
$$

Suppose that for $k-1$ we have $u_{k-1} \geq \alpha_{k-1} w_{k-1}, v_{k-1} \leq$ $\beta_{k-1} w_{k-1}$, and $\alpha_{k-2} \leq \alpha_{k-1} \leq \beta_{k-1} \leq \beta_{k-2}$. Then, for $k+1$, by the assumption $\left(H_{3}\right)$, we have

$$
\begin{aligned}
u_{k} & =A\left(u_{k-1}, v_{k-1}\right)=\int_{I} G\left(t, s, u_{k-1}(s), v_{k-1}(s)\right) d s \\
& \geq \int_{I} G\left(t, s, \alpha_{k-1} w_{k-1}, \beta_{k-1} w_{k-1}\right) d s \\
& \geq \phi\left(\alpha_{k-1}, \beta_{k-1}\right) \int_{I} G\left(t, s, w_{k-1}(s), w_{k-1}(s)\right) d s=\alpha_{k} w_{k}, \\
v_{k} & =A\left(v_{k-1}, u_{k-1}\right)=\int_{I} G\left(t, s, v_{k-1}(s), u_{k-1}(s)\right) d s \\
& \leq \int_{I} G\left(t, s, \beta_{k-1} w_{k-1}, \alpha_{k-1} w_{k-1}\right) d s \\
& \leq \varphi\left(\beta_{k-1}, \alpha_{k-1}\right) \int_{I} G\left(t, s, w_{k-1}(s), w_{k-1}(s)\right) d s=\beta_{k} w_{k} .
\end{aligned}
$$

By the above two inequalities and assumption $\left(\mathrm{H}_{3}\right)$, we have

$$
\begin{aligned}
\alpha_{k-1} & =\phi\left(\alpha_{k-2}, \beta_{k-2}\right) \leq \phi\left(\alpha_{k-1}, \beta_{k-1}\right)=\alpha_{k} \leq \beta_{k} \\
& =\varphi\left(\beta_{k-1}, \alpha_{k-1}\right) \leq \varphi\left(\beta_{k-2}, \alpha_{k-2}\right)=\beta_{k-1} .
\end{aligned}
$$

Then, it is easy to show by induction that inequalities $\left(11^{\prime}\right)$ and $\left(12^{\prime}\right)$ hold.

The following proof is similar to that of Theorem 4 . This completes the proof of Theorem 5 .

By a similar argument to that of Theorem 5, we obtain the following results.

Theorem 6. Let $P$ be a normal cone of $E$, and let $u_{0}, v_{0} \in P_{I}$ be coupled lower and upper quasi-solutions of (1). Assume that condition $\left(H_{1}\right),\left(H_{2}\right)$, and $\left(H_{3}\right)$ hold.

$\left(H_{4}^{\prime \prime}\right) G$ is $v_{0}$ quasi-lower, and there exists $w_{0} \in P_{I}$ such that $u_{0}<v_{0}<w_{0}$, and there exist $\alpha_{0}=\sup \left\{\alpha>0: u_{0} \geq\right.$ $\left.\alpha w_{0}\right\}, \beta_{0}=\inf \left\{\beta>0: v_{0} \leq \beta w_{0}\right\}$.

Then, (1) has a unique solution $x^{*}(t) \in D=\left[u_{0}, v_{0}\right]$, and for any initial $x_{0}, y_{0} \in\left[u_{0}, v_{0}\right]$, one has

$$
\begin{aligned}
& x_{n}(t) \longrightarrow x^{*}(t), \quad y_{n}(t) \longrightarrow x^{*}(t), \\
& \text { uniformly on } t \in I \text { as } n \longrightarrow \infty,
\end{aligned}
$$

where $\left\{x_{n}(t)\right\},\left\{y_{n}(t)\right\}$ are defined as

$$
x_{n}(t)=\int_{I} G\left(t, s, x_{n-1}(s), y_{n-1}(s)\right) d s,
$$

$$
y_{n}(t)=\int_{I} G\left(t, s, y_{n-1}(s), x_{n-1}(s)\right) d s, \quad t \in I \text {. }
$$




\section{Applications}

Consider the following two-point BVP in the Banach space:

$$
\begin{gathered}
-u^{\prime \prime}=f(t, u), \quad t \in J=[0,1], \\
u(0)=u(1)=0,
\end{gathered}
$$

where $f \in C[J \times P, P], P$ is a cone in a real Banach space $E$. Suppose that there exists a mapping $g \in C[J \times P \times P, P]$ such that $f(t, x)=g(t, x, x)$, and that $g$ satisfies the following conditions:

$\left(C_{1}\right) g$ is uniformly continuous on $J \times P \times P$, and $G$ is $\phi$ convex and $-\varphi$ concave quasi operator,

$\left(C_{2}\right) g(t, x, y)$ is nondecreasing in $x \in P$ for fixed $(t, y) \in$ $J \times P$, and $g(t, x, y)$ is nonincreasing in $y \in P$, for fixed $(t, x) \in J \times P$,

$\left(C_{3}\right)$ there exist the bounded nonnegative Lebesgue integrable functions $a(t), b(t), c(t)$, and $d(t)$ satisfying $\int_{J} a(s) d s<8, \int_{J} c(s) d s<8$ such that

$a(t) x+b(t) \leq g(t, x, y) \leq c(t) x+d(t), \quad t \in J, x, y \in P$.

It is well known that $u \in C^{2}[J, P]$ is a solution of $\operatorname{BVP}(34)$ in $C^{2}[J, P]$ if and only if $u \in C[J, P]$ is a solution of the following integral equation:

$$
u(t)=\int_{J} h(t, s) g(s, u(s), u(s)) d s, \quad t \in J,
$$

where

$$
h(t, s)= \begin{cases}t(1-s), & t \leq s, \\ s(1-t), & t>s .\end{cases}
$$

Lemma 7. If assumption $\left(C_{3}\right)$ holds, then there exists $u_{0}, v_{0} \in$ $C[J, P]$ such that

$$
\begin{aligned}
& u_{0}(t) \leq \int_{J} h(t, s) g\left(s, u_{0}(s), v_{0}(s)\right) d s, \quad t \in J, \\
& v_{0}(t) \geq \int_{J} h(t, s) g\left(s, v_{0}(s), u_{0}(s)\right) d s, \quad t \in J .
\end{aligned}
$$

Proof. In fact, let

$$
\begin{aligned}
& L_{1} u(t)=\int_{J} h(t, s) a(s) u(s) d s, \\
& x_{0}(t)=\int_{J} h(t, s) b(s) d s, \quad t \in J, \\
& L_{2} v(t)=\int_{J} h(t, s) c(s) v(s) d s, \\
& y_{0}(t)=\int_{J} h(t, s) d(s) d s, \quad t \in J .
\end{aligned}
$$

Obviously, by assumption $\left(C_{3}\right)$, we can get that $\left\|L_{1}\right\| \leq$ $\max _{t \in I}(t(1-t) / 2) \int_{J} a(s) d s=(1 / 8) \int_{J} a(s) d s<1$, then the equation $\left(I-L_{1}\right) u=x_{0}$ has a unique solution

$$
u_{0}(t)=\left(I-L_{1}\right)^{-1} x_{0}=\sum_{n=0}^{\infty} L_{1}^{n} x_{0} \in P_{I}
$$

Similarly, the equation $\left(I-L_{2}\right) v=y_{0}$ has a unique solution

$$
v_{0}(t)=\left(I-L_{2}\right)^{-1} y_{0}=\sum_{n=0}^{\infty} L_{2}^{n} y_{0} \in P_{I} .
$$

Thus, by assumption $\left(C_{3}\right)$, for any $t \in J$, we have

$$
\begin{gathered}
\int_{J} h(t, s) g\left(s, u_{0}(s), v_{0}(s)\right) d s \\
\geq \int_{J} h(t, s)(a(s) x+b(s)) d s \\
=L_{1} u_{0}(t)+x_{0}(t)=u_{0}(t), \\
\int_{J} h(t, s) g\left(s, v_{0}(s), u_{0}(s)\right) d s \\
\leq \int_{J} h(t, s)\left(c(s) v_{0}(s)+b(s)\right) d s \\
=L_{2} v_{0}(t)+y_{0}(t)=v_{0}(t),
\end{gathered}
$$

that is, (38) holds.

Theorem 8. Let $P$ be a normal cone of E. Assume that $\left(C_{1}\right)$ and $\left(C_{3}\right)$ hold,

$\left(C_{4}\right)$ there exists $w_{0} \in P_{I}$ and $u_{0}, v_{0}$ in (38) of Lemma 7 such that $u_{0}<w_{0}<v_{0}$, and also there exists $\alpha_{0}, \beta_{0} \in(0, \infty)$ such that $u_{0} \geq \alpha_{0} w_{0}, \beta_{0} w_{0} \geq v_{0}$,

$\left(C_{5}\right) \phi(\alpha, \beta), \varphi(\alpha, \beta)$ are all increasing in $\alpha$, decreasing in $\beta$ and $\phi\left(\alpha_{0}, \beta_{0}\right) \geq \alpha_{0}, \varphi\left(\beta_{0}, \alpha_{0}\right) \leq \beta_{0}$, for $\alpha, \beta \in$ $\left[\alpha_{0}, \beta_{0}\right], \alpha<\beta$,

$$
\varphi(\beta, \alpha)-\phi(\alpha, \beta) \leq l(\beta-\alpha), \quad 0<l<1 .
$$

Then, (34) has a unique solution $x^{*}(t) \in D=\left[u_{0}, v_{0}\right]$, and for any initial $x_{0}, y_{0} \in\left[u_{0}, v_{0}\right]$, one has

$$
\begin{aligned}
& x_{n}(t) \longrightarrow x^{*}(t), \quad y_{n}(t) \longrightarrow x^{*}(t), \\
& \text { uniformly on } t \in I \quad \text { as } n \longrightarrow \infty,
\end{aligned}
$$

where $\left\{x_{n}(t)\right\},\left\{y_{n}(t)\right\}$ are defined as

$$
\begin{gathered}
x_{n}(t)=\int_{J} h(t, s) g\left(s, x_{n-1}(s), y_{n-1}(s)\right) d s, \\
y_{n}(t)=\int_{J} h(t, s) g\left(s, y_{n-1}(s), x_{n-1}(s)\right) d s, \quad t \in J .
\end{gathered}
$$


Proof. It is easy to see by conditions $\left(C_{1}\right)$ and $\left(C_{2}\right)$ that $G(t, s, x, y)=h(t, s) g(s, x, y)$ satisfy the conditions $\left(H_{1}\right)$ and $\left(H_{2}\right)$ of Theorem 4. By $\left(C_{3}\right)$ and (38), we have $u_{0}, v_{0} \in C[I, P]$ as coupled lower and upper quasi-solutions of (34).

Thus, the assumption $\left(H_{1}\right)-\left(H_{4}\right)$ of Theorem 4 is satisfied from the assumption $\left(C_{1}\right)-\left(C_{5}\right)$ of Theorem 8 . The conclusion of Theorem 8 follows from Theorem 4 .

Example 9. In fact, we can construct the function $f(t, x)$ in Theorem 8.

Let

$$
\begin{aligned}
& f(t, x)= g(t, x, y)=x+\frac{1}{y}, \quad t \in[0,1], \\
& \phi(\alpha, \beta)=\sin \alpha+\frac{1}{2 \beta}, \\
& \alpha \in\left[0, \frac{\pi}{2}\right], \\
& \varphi(\alpha, \beta)=3 \alpha-5 \beta,
\end{aligned}
$$

then

$$
\begin{aligned}
G(t, s, \alpha x, \beta y)=h(t, s) g(s, \alpha x, \beta y) \\
=h(t, s)\left(\alpha x+\frac{1}{\beta y}\right) \\
\quad \geq h(t, s)\left(\sin \alpha+\frac{1}{2 \beta}\right)\left(x+\frac{1}{y}\right) \\
=\phi(\alpha, \beta) G(t, s, x, y), \\
G(t, s, \alpha x, \beta y)=h(t, s) g(s, \alpha x, \beta y) \\
=h(t, s)\left(\alpha x+\frac{1}{\beta y}\right) \\
\quad \leq h(t, s)(3 \alpha-5 \beta)\left(x+\frac{1}{y}\right) \\
=\varphi(\alpha, \beta) G(t, s, x, y) .
\end{aligned}
$$

Thus, $G$ is $\phi$ convex and $-\varphi$ concave quasi operator and thus satisfies $\left(C_{1}\right)$.

It is easy to check that $g(t, x, y)$ is nondecreasing in $x$ for fixed $(t, y)$ and is nonincreasing in $y$ for fixed $(t, x)$ and thus satisfies $\left(C_{2}\right)$.

There exist $a(t)=t / 2, b(t)=t / 100, c(t)=2 t$, and $d(t)=1000 t$ satisfying

$$
\begin{aligned}
& \int_{0}^{1} a(s) d s=\frac{1}{2} \int_{0}^{1} t d t=\frac{1}{4}<8 \\
& \int_{0}^{1} c(s) d s=2 \int_{0}^{1} s d s=1<8
\end{aligned}
$$

such that

$$
a(t) x+b(t) \leq g(t, x, y) \leq c(t) x+d(t) .
$$

Thus, $\left(C_{3}\right)$ holds.
There exist

$$
\begin{gathered}
u_{0}=\int_{0}^{1} h(t, s)\left[u_{0}(s)+\frac{1}{v_{0}(s)}\right] d s, \\
v_{0}=2 u_{0}=\int_{0}^{1} h(t, s)\left[v_{0}(s)+\frac{1}{u_{0}(s)}\right] d s .
\end{gathered}
$$

Choose $w_{0}=(3 / 2) u_{0}$ such that $u_{0}<w_{0}<v_{0}$, and also there exist $\alpha_{0}=2 / 3, \beta_{0}=4 / 3$ such that

$$
u_{0}=\alpha_{0} \frac{3}{2} u_{0}=\alpha_{0} w_{0}, \quad \beta_{0} \frac{3}{2} u_{0}=2 u_{0}=v_{0} .
$$

Thus, $\left(C_{4}\right)$ is satisfied.

$\phi(\alpha, \beta), \varphi(\alpha, \beta)$ are all increasing in $\alpha$ and nondecreasing in $\beta$,

$$
\begin{aligned}
& \phi\left(\frac{2}{3}, \frac{4}{3}\right)=\sin \frac{2}{3}+\frac{1}{2 \times(4 / 3)} \geq \frac{2}{3}=\alpha_{0} \\
& \varphi\left(\frac{4}{3}, \frac{2}{3}\right)=3 \times \frac{4}{3}-5 \times \frac{2}{3}=\frac{2}{3} \leq \frac{4}{3}=\beta_{0}
\end{aligned}
$$

and for $\alpha, \beta \in[2 / 3,4 / 3], \alpha<\beta$, we have

$$
\varphi(\beta, \alpha)-\phi(\alpha, \beta)=3 \beta-5 \alpha-\sin \alpha-\frac{1}{2 \beta} \leq \frac{99}{100}(\beta-\alpha) \text {. }
$$

Thus, $\left(C_{5}\right)$ also holds.

\section{Acknowledgment}

The author was supported financially by Natural Science Foundation of China (11071141) and the Project of Shandong Province Higher Educational Science and Technology Program (J13LI12, J10LA62).

\section{References}

[1] D. Guo and V. Lakshmikantham, "Coupled fixed points of nonlinear operators with applications," Nonlinear Analysis, vol. 11, no. 5, pp. 623-632, 1987.

[2] D. Guo, "Extremal solutions of nonlinear Fredholm integral equations in ordered Banach spaces," Northeastern Mathematical Journal, vol. 7, no. 4, pp. 416-423, 1991.

[3] S. Jingxian and L. Lishan, "Iterative method for coupled quasisolutions of mixed monotone operator equations," Applied Mathematics and Computation, vol. 52, no. 2-3, pp. 301-308, 1992.

[4] L. Lishan, "Iterative method for solutions and coupled quasisolutions of nonlinear Fredholm integral equations in ordered Banach spaces," Indian Journal of Pure and Applied Mathematics, vol. 27, no. 10, pp. 959-972, 1996.

[5] Z. T. Zhang, "The fixed point theorem of mixed monotone operator and applications," Acta Mathematica Sinica, vol. 41, no. 6, pp. 1121-1126, 1998 (Chinese).

[6] L. S. Liu, "Iterative solutions of nonlinear mixed monotone Hammerstein type integral equations in Banach spaces," Journal of Systems Science and Mathematical Sciences, vol. 16, no. 4, pp. 338-343, 1996 (Chinese). 
[7] H. Su and L. S. Liu, "Iterative solution for systems of a class abstract operator equations and applications," Acta Mathematica Scientia A, vol. 27, no. 3, pp. 449-455, 2007 (Chinese).

[8] Y. Liu and A. Qi, "Positive solutions of nonlinear singular boundary value problem in abstract space," Computers and Mathematics with Applications, vol. 47, no. 4-5, pp. 683-688, 2004.

[9] Y. Wu, "New fixed point theorems and applications of mixed monotone operator," Journal of Mathematical Analysis and Applications, vol. 341, no. 2, pp. 883-893, 2008.

[10] Z. Zhao and X. Du, "Fixed points of generalized e-concave (generalized e-convex) operators and their applications," Journal of Mathematical Analysis and Applications, vol. 334, no. 2, pp. 1426-1438, 2007.

[11] Z. Zhao, "Existence and uniqueness of fixed points for some mixed monotone operators," Nonlinear Analysis, Theory, Methods and Applications, vol. 73, no. 6, pp. 1481-1490, 2010.

[12] X. Zhang, L. Liu, and Y. Wu, "Global solutions of nonlinear second-order impulsive integro-differential equations of mixed type in Banach spaces," Nonlinear Analysis, Theory, Methods and Applications, vol. 67, no. 8, pp. 2335-2349, 2007.

[13] Z. Zhao and X. Zhang, "C (I) Positive solutions of nonlinear singular differential equations for nonmonotonic function terms," Nonlinear Analysis, Theory, Methods and Applications, vol. 66, no. 1, pp. 22-37, 2007.

[14] D. Guo, "Initial value problems for second-order integrodifferential equations in Banach spaces," Nonlinear Analysis, Theory, Methods and Applications, vol. 37, no. 3, pp. 289-300, 1999. 


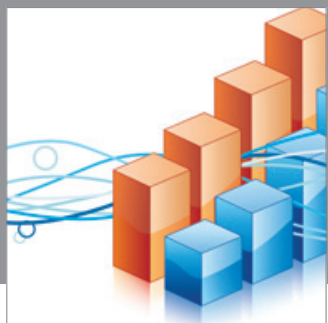

Advances in

Operations Research

mansans

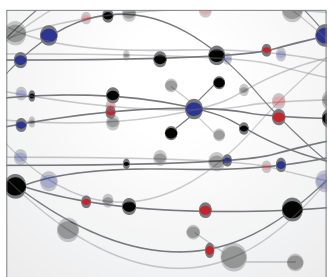

The Scientific World Journal
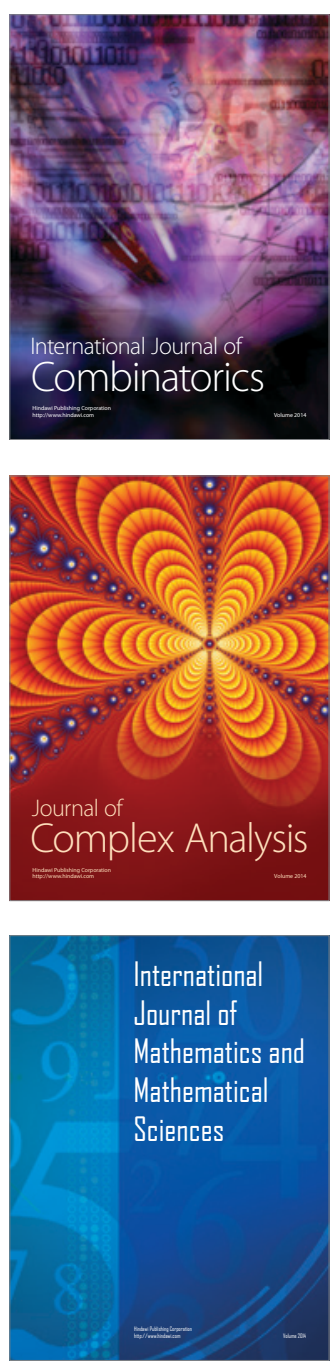
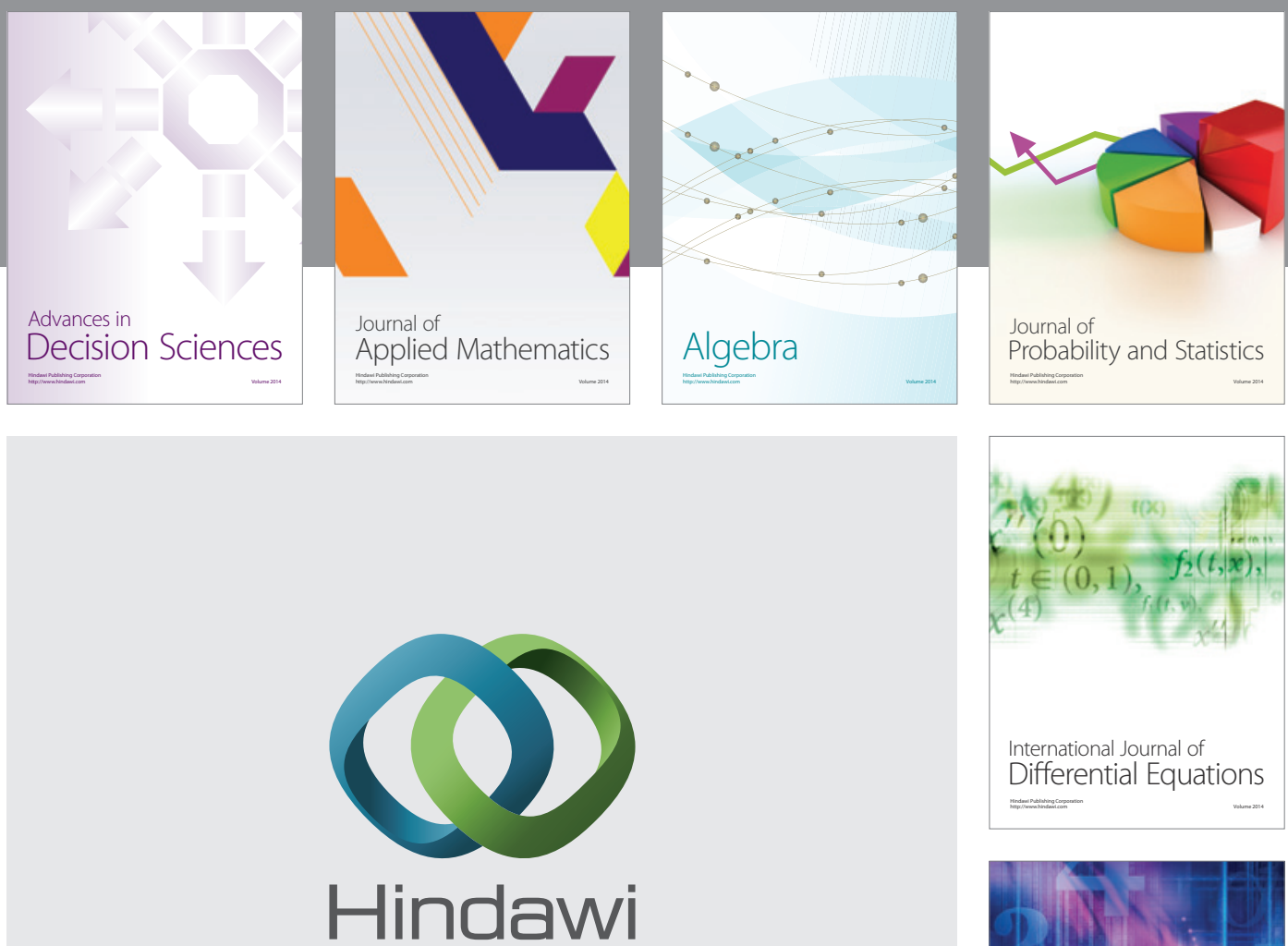

Submit your manuscripts at http://www.hindawi.com
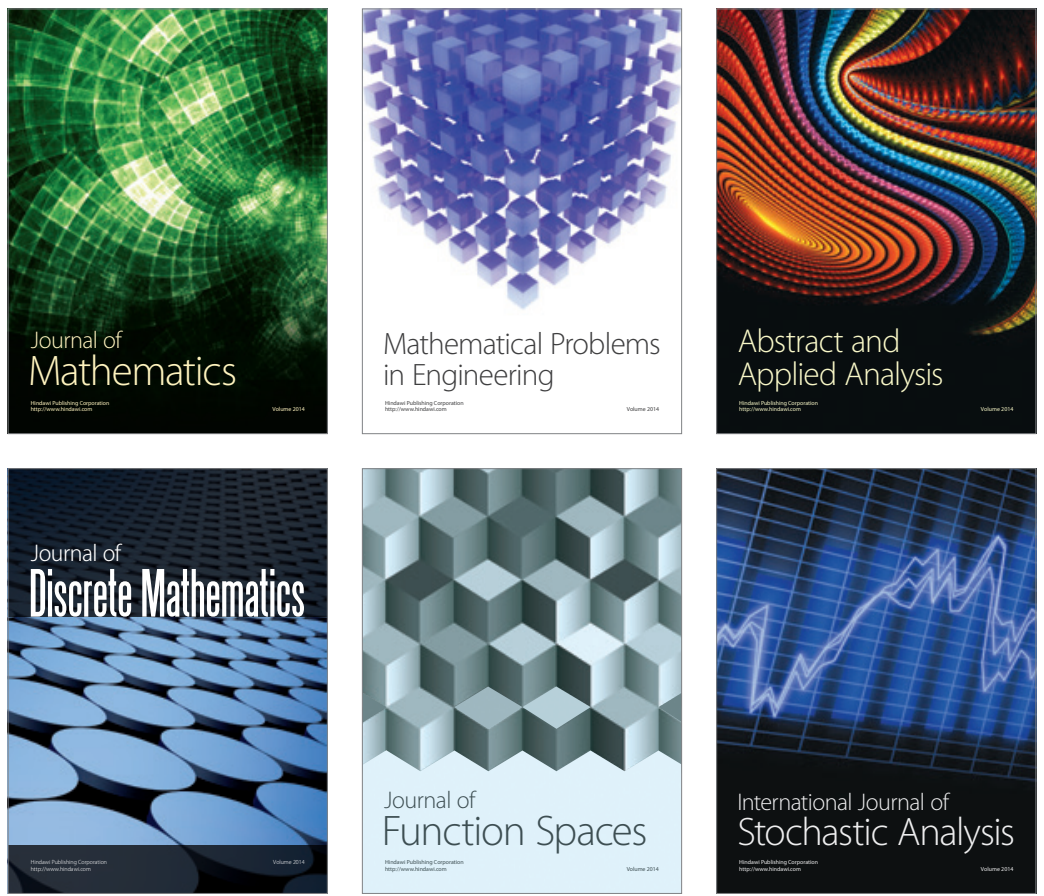

Journal of

Function Spaces

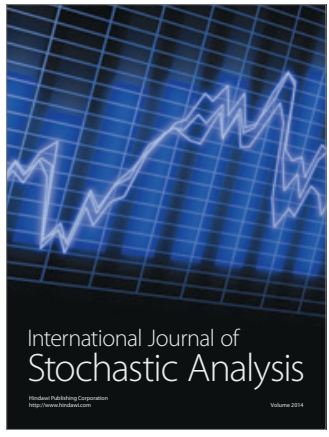

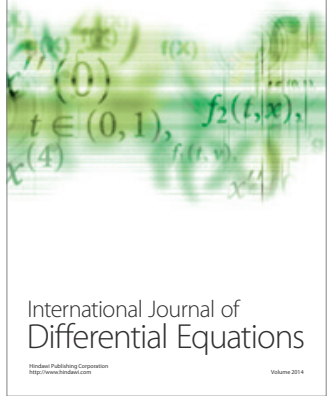
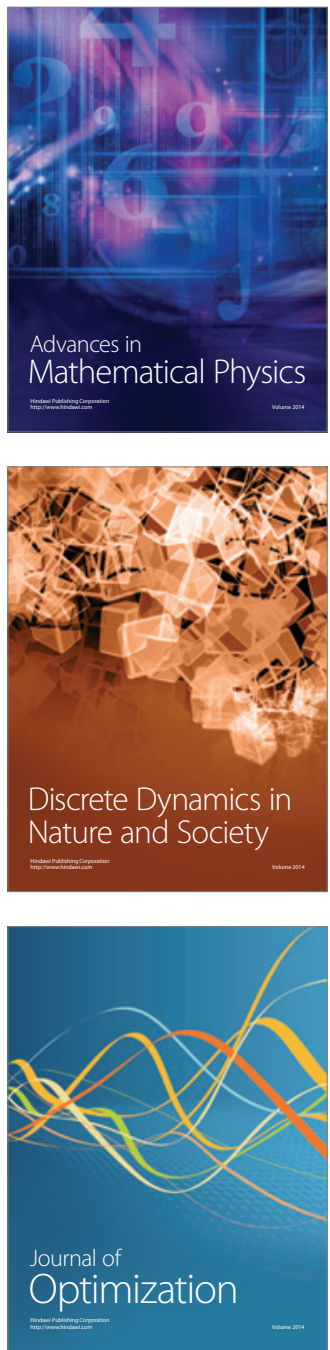